\title{
Learning from the wastage: histomorphology of columnar cell lesions and other changes in surgical pathology breast specimens
}

\author{
Dharitri Bhat ${ }^{1, *}$, Poonam A. Malani ${ }^{2}$, Dinkar T. Kumbhalkar ${ }^{3}$ \\ ${ }^{1}$ Associate Professor, ${ }^{2}$ Senior Resident, ${ }^{3}$ Professor, Dept. of Pathology, Government Medical College, Nagpur, Maharashtra, \\ India
}

*Corresponding Author:

Email: dharitribhat@gmail.com

\begin{abstract}
Introduction: In recent few decades, widespread use of mammography has resulted in increased detection of precursor and preinvasive breast lesions such as atypical ductal hyperplasia, ductal carcinoma in situ and, a type of breast lesion termed, 'Flat epithelial atypia" which belongs to the group of columnar cell lesions with atypia. Although in India, invasive breast cancer is still most common presentation than early preinvasive lesions, with advanced diagnostic set up and increased awareness, early detection of preinvasive lesions is bound to be increased in future. For their accurate diagnosis, it is very necessary to be aware and alert about the morphology of these lesions.

Objectives: Present study was undertaken to study the morphology of Columnar Cell Lesion and other changes in Surgical Biopsy specimens received in Histopathology laboratory of a tertiary health care centre.

Materials and Methods: Excessive sections were taken from breast tissue surrounding the primary lesion in mastectomy or wide local excision specimens received over two-year duration and were examined for various changes in the epithelium and in the stroma especially columnar cell lesions and other precursor lesions.

Results: A spectrum of precursor lesions including columnar cell lesions could be commonly seen in most of the specimens. Their morphology and differential diagnosis could be studied in details in these sections which otherwise are wasted usually.

Conclusion: The tissue surrounding primary lesion in surgical pathology breast specimens is a good source to study the detailed morphology of columnar cell lesions and other lesions to get familiar with them for their accurate detection and early diagnosis in future.
\end{abstract}

Keywords: Precursor breast lesions, Columnar cell lesions, Flat epithelial atypia, Ductal hyperplasia.

\section{Introduction}

Invasive breast cancer is more than twice as common as cancer at any other site in women. 1 Currently, the focus on the breast cancer has shifted from treatment to early diagnosis which is associated with dramatic improvement in survival. ${ }^{2}$ In recent few decades, widespread use of mammography has resulted in increased detection of precursor and preinvasive breast lesions such as atypical ductal hyperplasia and ductal carcinoma in situ and certain lesions such as "Flat Epithelial Atypia" (FEA)which has been included in DIN (Ductal Intraepithelial Neoplasia) system by WHO in 2003 as (DIN Ia). ${ }^{3}$ Flat Epithelial Atypia includes columnar cell lesions (CCL)with atypia which, can be easily missed on low power examination due to its subtle morphological changes. Such lesions are currently been encountered with increasing frequency in breast biopsies done for abnormal mammographic findings specially microcalcifications. Unfortunately, in India, most of the cases of breast cancer still present in advanced stage and early detection is yet to become effective like western countries. With increase in awareness and preventive oncology set up, it is bound to be encountered with greater frequency in future. The responsibility for early detection will rest heavily on the pathologists and may cause diagnostic difficulty. So, it is very necessary to be aware and alert about the morphology of these lesions for diagnosing them accurately. Currently the best way to learn morphology of these lesions is to examine various surgical pathology breast specimens which often show these lesions in the surrounding breast tissue which is mostly left over except few sections. Present study is aimed at studying the morphology of various precursor lesions specially the spectrum of columnar cell lesions, so that their identification in future becomes easier and accurate.

\section{Aims and Objectives}

Present study is carried out in The Department of Pathology of Government Medical College with following aims and objectives:

1. To study the morphology of columnar cell lesions and to categorize various columnar cell lesions in tissue surrounding the primary lesion of surgical pathology breast specimens.

2. To find other histopathological changes in the surrounding breast tissue in surgical pathology breast specimens.

\section{Materials and Methods}

Present study is a prospective observational case study of 2 years duration carried out in the pathology department of Government Medical College Nagpur (a tertiary health care centre). This included the surgical 
breast specimens received in histopathology section of the department over two years.

Inclusion Criteria: All the specimens showing some amount of grossly uninvolved breast tissue apart from the primary lesion for which the surgery was performed were included in the study.

\section{Exclusion Criteria:}

1. The specimens like lumpectomy and excisional biopsy showing only primary pathology (for which the surgery was performed) and no apparently normal breast tissue adjacent to the lesion were not included in the study.

2. The lesions associated with dense inflammation and exudate masking the cellular details in the adjacent breast tissue, were also not included in the study.

The types of specimens included in the study were:

1. Simple mastectomy (SM)

2. Radical and modified radical mastectomy (MRM)

3. Breast conserving surgery (Wide local excision WLE or specimen with adjacent breast tissue) At the time of receiving each specimen, complete clinical details including past and family history, menstrual and obstetric history, general and local examination findings such as (size, consistency, mobility, nipple discharge, overlying skin, lymph node status) and investigations done (FNAC, Core Needle Biopsy(CNB), lumpectomy, ultrasonography or mammography) were also noted. The specimen examination included nature of the specimen, size, shape, overlying skin changes, cut surface findings like size and location of the lump, colour, consistency, margins, and fixity to other structures, cystic changes, presence of haemorrhages and necrosis, surrounding breast tissue status and number of lymph nodes identified.

Sectioning: After routine sectioning for regular histopathology reporting, additional sections were taken varying on the type of specimen. Total 6 to 8 sections were taken in addition to routine sections from the lesions. (Fig. 1 showing the method of taking extra sections from the surrounding breast tissue.) ${ }^{4}$ After routine tissue processing, $H \& E$ sections were viewed for different histopathological findings and columnar cell lesions in particular.

\section{Observations}

This study included total 157 cases, of which one was a male patient. Youngest patient was 22 years female with diagnosis of Phyllodes tumour and eldest patient was 87 years with Infiltrating Duct Carcinoma (IDC). Total 138 specimens were of mastectomy (MRM), 7 simple mastectomy and 12 cases of wide local excision (WLE). MRM cases were all of different types of malignancies such as IDC and its variants, Invasive Lobular Carcinomas (ILC) and other types such as metaplastic carcinoma. Simple mastectomy cases included IDC (3), metaplastic carcinoma (2) and myoepithelial carcinoma (1). WLE included the cases of benign phyllodes tumour (5), malignant phyllodes tumour (2), ductal carcinoma in situ (DCIS)-(3) stromal sarcoma (1) and giant fibroadenoma (1) which was diagnosed as phyllodes tumour on FNAC. Table 1 shows the list of diagnosis seen in this study.

Of the total 157 specimens, surrounding sections from 128 cases $(82 \%)$ showed various changes. No changes were seen in $29(18 \%)$ cases. Few cases showed changes in the epithelium as well as in the stroma. Table 2 shows various epithelial changes seen in the study. It included proliferative as well as nonproliferative changes. Table 3 lists various stromal and other changes seen in all cases. Apart from epithelial and stromal changes, other changes included lymphovascular emboli and invasion in 12(7.64\%), Periductal Inflammation 20(12.73\%), Giant Cell Reaction 7 (4.45\%), calcification $9(5.73 \%)$, medial calcification of vessels $8(5.09 \%)$, granulation tissue $2(1.27 \%)$ and Thrombosed vessels $1(0.63 \%)$ cases. Morphology of CCL was studied in details in order to categorize them as per DIN system. The classification system by Rosen et al was used. ${ }^{5}$ Table 4 shows the split up of cases of CCLwith and without Atypia.

\section{Discussion}

Present observational case study entitled "Learning from the wastage: Histomorphology of Columnar Cell Lesions and other changes in Surgical Pathology Breast Specimens" carried out at a tertiary Health care centre included 157 breast specimens with varied diagnosis. In all cases, sections from surrounding tissue were examined for columnar cell lesions and other changes seen in epithelium as well as stroma. Most important feature in this study was morphology of Columnar Cell Lesions with and without Atypia. Although first recognized by John Collins Warren in 1905, CCL have been described by a number of authors by various terminologies. The concept of 'clinging carcinoma in situ' was introduced by Azzopardi in 1979 to describe a distinct atypical intraepithelial lesion of the breast that can be mistaken for either normal breast tissue or ordinary fibrocystic breast change. The morphological comparison of these lesions with more conventional type of DCIS, particularly the comparison of cytological features of these lesions at higher magnification convinced Azzopardi that these must reflect another type of in situ ductal carcinoma. It was a descriptive term for another type of in situ cancer characterized by only 1,2, or just a few layers of atypical cells lining the structure of origin- hence clinging in the sense that malignant (atypical) cells are present peripherally while there is no significant intraluminal proliferation. ${ }^{5,6}$ Since then, many pathologists have found an association between the lesions with such pattern of growth and invasive breast cancer. Finally, after more than 20 years, a long period in which the lesion remained widely unrecognized and /or ignored, the most recent WHO Classification of 
Tumors of the Breast and Female Genital Organs (2003) acknowledged the neoplastic nature of atypical epithelial cells in this variant of intraepithelial breast lesions and accepted the designation of Flat epithelial atypia or flat DIN, ductal intraepithelial neoplasia grade Ia (DIN Ia), for those lesions associated with mild nuclear atypia. Despite the diversity of published descriptions and the wide assortment of names applied to these breast lesions, for practical purpose these lesions can be grouped into two broad diagnostic categories, columnar cell changes and columnar cell hyperplasia. ${ }^{7}$ Both of which can be further subdivided into two based on with or without atypia. The category of CCL with atypia (CCC with atypia) and $\mathrm{CCH}$ with atypia are collectively included under the term Flat epithelial Atypia. $^{8-11}$

\section{Morphology of CCL}

1. CCC: Variably dilated acini lined by one or 2 layers of columnar cells with regular size and shape, bland nuclei perpendicular to the basement membrane, occasional apical snouts and intraluminal secretions.

2. CCH: Similar to CCC with focal stratification and multilayering with focal nuclear crowding and overlapping and variable nuclear hyperchromasia.

3. CCL- (CCC or CCH with Atypia FEA): A ductal epithelial proliferation along the entire perimeter with low- grade cytological atypia in the absence of architectural atypia. Since the lesions with atypia have been proved to be a precursor for invasive breast cancer, identification of these lesions in CNB is very important.

Of the total 157 cases included in the study, one was male patient who underwent MRM for IDC. Youngest patient in this study was 22 years old with benign phyllodes tumour, eldest patient was of 87 years old with IDC. Maximum patients belonged to age group between 41 to 50 years (49). Total 138 specimens were of mastectomy (MRM), 7 simple mastectomy and 12 cases of wide local excision. Most frequently encountered diagnosis was Infiltrating Duct Carcinoma Not Otherwise Specified (IDC NOS)- (122). Other diagnoses were Infiltrating Lobular Carcinoma - ILC (6), Benign (5) and malignant (2) phyllodes tumour, Medullary carcinoma (4), Metaplastic Carcinoma (3), Tubular Carcinoma (2), and one case each of Stromal sarcoma, Myoepithelial carcinoma, Papillary carcinoma, and Giant fibroadenoma. In all cases, primary histopathological diagnosis was based on either FNAC, or histopathology (CNB or excisional biopsy). Wide local excision (12) specimens included cases of Phyllodes tumour (benign 5 \& malignant 2) and 3 cases of DCIS, one case each of stromal sarcoma, and Giant fibroadenoma. In case of Giant fibroadenoma, primary diagnosis was phyllodes tumour on FNAC hence wide local excision was done, which on histopathology turned out to be Giant fibroadenoma. In 3 cases, FNAC diagnosis was Proliferative Breast Disease with Atypia, clinical diagnosis was fibroadenoma; so wide local excision was done, which on histopathology showed foci of DCIS. In 6 mastectomy specimens, histological diagnosis was given as DCIS, as no focus of IDC was found even on thorough sampling of the tumour. In all these cases, history of prior excision or lumpectomy was obtained indicating removal of the invasive focus; and the residual mastectomy specimens showed only focus of DCIS. Simple mastectomy specimens (7) included 4 cases of IDC, 2 cases of metaplastic carcinoma and 1 case of myoepithelial carcinoma.

In this study, $128 / 157$ cases $(81.52 \%)$ cases showed changes in surrounding tissue, including epithelial, stromal and other changes. 29 cases (18.47\%) showed no changes i. e, normal surrounding tissue. MRM specimen from male breast showed no change in adjacent tissue. Allred et al in their study on Premalignant breast disease stated the possibility that some IBCs arise directly from morphologically normal appearing cells. ${ }^{12}$ Sections from surrounding tissue showed changes in the epithelium, (106/157-67.51\%), stroma (35/157 - $22.29 \%$ cases), and other changes in $(55 / 157-35.03 \%)$ cases. There are plenty of studies on morphology of columnar cell Lesions with and without atypia along with their historical aspects, and clinical significance based on molecular and genetic studies. Most of the studies are on CNB done for mammographically detected abnormal calcifications in high risk patients or during routine screening or follow up studies. We could not get any similar study on morphology of columnar cell lesions and other changes in adjacent tissue in surgically excised breast specimens.

Epithelial changes: Epithelial changes are categorized into two, proliferative and non- proliferative. All types of hyperplasia with at least some increase in the risk of invasive breast cancer are included in proliferative lesions; remaining epithelial lesions are nonproliferative. ${ }^{13}$ Out of total 157 cases included in the study, FEA was seen in 68 cases $(43.31 \%)$. These lesions were often seen coexistent with other columnar lesions without atypia $(\mathrm{CCC}, \mathrm{CCH})$. Morphological diversity in the form of variation in nuclear staining intensity, shape and tallness of the cell was also noted as mentioned by Walker RA et al. ${ }^{11}$ Although the cells lining the TDLU in FEA are usually columnar, many times cuboidal cells were also seen, and as mentioned by Schnitt \& O'Malley. ${ }^{10,12}$ It is the cytological atypia of low grade that should be kept in mind while diagnosing FEA. ${ }^{14}$ (Fig. 2c.d) With increase in the numbers of layers, adjacent foci of ADH and DCIS were also seen which might be indicating that it is a continuum of process. Occasional appearance of mitosis was also noted in these lesions along with presence of intraluminal secretions including necrosis and calcifications. All other intraductal proliferations like Usual Ductal Hyperplasia (UDH, Atypical Ductal Hyperplasia (ADH) and DCIS are usually suspected on 
low power examination due to their characteristic architectural appearance. (Fig. 3). In FEA, however, no such architectural change is seen and can be easily missed in low power or scanner view and the diagnosis is based on nuclear findings in high power examination..$^{8,9,15}$ Other features that were commonly seen in these cases of FEA were variable degree of acinar dilatation, cell crowding and overlapping, and formation of apical snouts. (Fig 2) Pathmanathan $\mathrm{N}$ et al used a scoring system for classification of these lesions along with immunohistochemical profile for ER and $\mathrm{Ki} 67 .{ }^{16}$ In present study, 16 cases $(10.19 \%)$, CCLs were seen without atypia; whereas in cases of FEA, coexistence of CCC and CCL was very common. Turashvilli's $\mathrm{G}$ et al in their study on association of quantitative breast histology with mammographic density in forensic autopsy series gave detailed description of the morphological criteria to differentiate CCL with and without atypia and mentioned that in atypical lesions, nuclei are more round to oval than elongated and are not oriented perpendicularly to the basement membrane, show increased nucleocytoplasmic ratio, evenly dispersed or slightly marginated nuclear chromatin and variably prominent nucleoli..$^{5}$ These findings were helpful for us in morphological categorization of these lesions.

Differential Diagnosis of CCLs/ FEA: Various lesions that can be confused with CCLs on morphology are Microcysts in Fibrocystic disease, Apocrine cystic change and Blunt duct adenosis. (Fig 4) Microcysts in Fibrocystic Disease show Flattened lining epithelium of the dilated glandular spaces. Apocrine Cystic Change show characteristic dense granular eosinophilic cytoplasm which is more voluminous than columnar cells in FEA, and usually round nuclei with open chromatin and prominent nucleoli. In contrast to this in FEA, the nuclei are more hyperchromatic with powdery chromatin and variable degree of atypia depending on the grade. ${ }^{14,16}$ These features were helpful in differentiating FEA from apocrine cysts. In our study, apocrine change was seen in 8 cases without any diagnostic difficulty. Of these 8 cases, 6 were IDC, one was DCIS and one case was of metaplastic carcinoma. Blunt Duct Adenosis: Differentiating FEA from BDA was more difficult than apocrine change. Although there are well defined criteria for the diagnosis of BDA; in few cases applying them on sections was difficult. The main reason for this may be that BDA also shows columnar alteration of cells along with dilatation of glands. ${ }^{13,17}$ The morphological features that helped us in differentiating these lesions from FEA were 1. Tubular or branching shapes seen in BDA in contrast to globoid cystic glands seen in FEA. ${ }^{2}$ Hypertrophy of the myoepithelial cells seen as prominent continuous ring around the gland and 3. Expanded, cellular intralobular stroma in contrast to diminished stroma in FEA. (Fig. 4) We found 8 cases of BDA, (IDC-7) and one case with metaplastic carcinoma. Low power or scanner view examination is very helpful in diagnosing these lesions, specially the contour of the dilated glands.

Usual Ductal Hyperplasia: In Our study, foci with UDH were seen in 20 cases $(12.73 \%)$ which included 16 cases of IDC, 2 DCIS and one each of ILC and metaplastic carcinoma. At times, UDH is known to be confused with CCL. The cells in UDH show variation in cell size and shape so that the cells in the centre are smaller than that in the periphery along the basement membrane. Even though some nuclei are larger, there is no hyperchromasia. We found the admixture of myoepithelial cells and peripheral slit like spaces as the most helpful finding in diagnosing UDH. Formation of micro papillae in ductular spaces within UDH often creates confusion as it may resemble focus of $\mathrm{ADH}$ or DCIS. Coexistence of these foci with FEA and ADH and even DCIS was also seen. As said by Moinfar F, the use of standardized criteria for the diagnosis and uniform terminology is essential in establishing the significance and determining the guidelines for the management of these lesions. For this, studies with more numbers of patients that allow reliable statistical analysis are needed. ${ }^{3}$

ADH: We came across with 7 cases of ADH (3 in IDC, 2 with DCIS and 2 with ILC). Presence of architectural atypia is essential for the diagnosis of ADH. It is more difficult to distinguish ADH from DCIS. Since FEA often merges into ADH or DCIS, sometimes overlapping features were seen. Foci of ADH are often known to coexist with DCIS and FEA hence the diagnosis is always difficult. The flat growth pattern seen in FEA along the entire perimeter of the involved gland without formation of papillae, bridges, arches or cribriform spaces often favours the diagnosis of FEA. ${ }^{18}$ Adjacent areas often showed coexistent foci similar to ADH or more commonly DCIS. For the diagnosis of $\mathrm{ADH}$, strict qualitative as well as quantitative criteria need to be fulfilled. ADH is usually a small and focal measuring less than 2 to $3 \mathrm{~mm}$, it is rare and seen in $4 \%$ of symptomatic benign biopsies. ${ }^{19,20}$ In DIN terminology $\mathrm{ADH}$ is termed as DIN Ib, The WHO recommends that for purposes of clinical management and tumor registry coding, when the DIN terminology is used, the traditional terminology should be mentioned as well. ${ }^{1} \mathrm{CGH}$ analysis on ADH and DCIS support the concept that LG-DCIS and HG- DCIS are likely to arise from two distinct evolutionary pathways and that $\mathrm{ADH}$ and FEA shares many alterations with LG-DCIS and LG -IDC. ${ }^{20}$ FEA is genetically related to $\mathrm{ADH}$ and is a likely precursor to $\mathrm{ADH} .^{21}$

DCIS: In Adjacent tissue, we found DCIS areas in 27 cases (IDC-23, ILC-2, PC and Med C 1 each) Many studies advocate subtyping of DCIS into Low grade and High Grade based on cytonuclear features. According to some, intermediate grade is also included in classification. ${ }^{1,15,18,22}$

Lobular Hyperplasia and Neoplasia: We found lobular hyperplasia and atypical lobular hyperplasia in 
28 cases and LCIS in 6 cases. There can be overlap with lobular hyperplasia and LCIS. In ALH, quantitative criteria are more important i.e., cells identical in appearance as in LCIS but involving less than one half of the acinar units. In lobular neoplasia, the nuclei are pale and round. Cytoplasmic vacuoles or clear cytoplasm was very important diagnostic clue in differentiating these lesions from ductal. LCIS lesions can also be confused with solid type of DCIS. Formation of well-formed spaces and or intraluminal necrosis favoured the diagnosis of DCIS. Use of special stains like PAS or Alcian Blue can prove helpful. Other changes included sclerosing adenosis and periductal inflammation . $^{711,13,15}$
Stromal Changes: In present study, changes in the stroma were seen in $35 / 157$ cases. Table 4 shows the stromal and other changes. Most common change in the stroma was hyalinization, seen in $25(15.92 \%)$ cases, elastosis 5 (3.18 \%), and PASH like stroma $3(1.91 \%$ $\%)$, Fat necrosis $3(1.91 \%)$. Other changes were calcification in the duct lumen, in the vessel walls (medial calcification and also in the stroma, stromal edema and haemorrhages with hemosiderin laden macrophages. Foci of lympho-vascular invasion were also seen. Sections from breast tissue surrounding the lesion in surgical breast specimens form the best source to study the morphology of various lesions specially those with diagnostic significance.

Table 1: Showing list of all diagnosis included in the study

\begin{tabular}{|c|l|c|}
\hline S. No. & \multicolumn{1}{|c|}{ List of Diagnosis } & No. of cases \\
\hline 1 & Infiltrating Duct Carcinoma & 122 \\
\hline 2 & Infiltrating Lobular Carcinoma & 6 \\
\hline 3 & Ductal Carcinoma in Situ & 9 \\
\hline 4 & Benign Phyllodes Tumour & 5 \\
\hline 5 & Medullary Carcinoma & 4 \\
\hline 6 & Metaplastic Carcinoma & 3 \\
\hline 7 & Malignant Phyllodes Tumor & 2 \\
\hline 8 & Tubular Carcinoma & 2 \\
\hline 9 & Papillary Carcinoma & 1 \\
\hline 10 & Stromal Sarcoma & 1 \\
\hline 11 & Myoepithelial Carcinoma & 1 \\
\hline 12 & Giant fibroadenoma & 1 \\
\hline & Total & 157 \\
\hline
\end{tabular}

Table 2: Showing proliferative epithelial changes seen in this study

\begin{tabular}{|c|l|c|}
\hline S. No. & \multicolumn{1}{|c|}{$\begin{array}{c}\text { Epithelial Changes Seen } \\
\text { (Proliferative) }\end{array}$} & $\begin{array}{c}\text { No. of } \\
\text { Cases/ 157 }\end{array}$ \\
\hline 1 & Flat Epithelial Atypia & $68(43.31 \%)$ \\
\hline 2. & $\begin{array}{l}\text { Columnar cell Lesions without } \\
\text { Atypia }\end{array}$ & $16(10.19 \%)$ \\
\hline 3 & Usual ductal Hyperplasia & $20(12.73 \%)$ \\
\hline 4 & Atypical Ductal Hyperplasia & $7(4.45 \%)$ \\
\hline 5 & Ductal Carcinoma In Situ & $27(17.19 \%)$ \\
\hline 6 & Lobular Carcinoma in Situ & $6(3.82 \%)$ \\
\hline 7 & Lobular Hyperplasia / Atypical LH & $28(17.83 \%)$ \\
\hline 8 & Micropapillary Hyperplasia & $6(3.82 \%)$ \\
\hline 9 & Blunt Duct Adenosis & $8(5.09 \%)$ \\
\hline
\end{tabular}

Table 3: Showing Non-proliferative epithelial changes in the study

\begin{tabular}{|c|l|c|}
\hline S. No. & Epithelial Changes & No. of cases /157 \\
\hline 1 & Fibrocystic changes & $18(11.46 \%)$ \\
\hline 2 & Apocrine change & $8(5.09 \%)$ \\
\hline 3 & Sclerosing Adenosis & $7(4.45 \%)$ \\
\hline 4 & Duct Ectasia & $5(3.18 \%)$ \\
\hline 5 & Lactational Change & $1(0.63 \%)$ \\
\hline 6 & Cancerization of the Lobule & $1(0.63 \%)$ \\
\hline
\end{tabular}


Table 4: Showing stromal and other changes seen in the study

\begin{tabular}{|c|l|c|}
\hline S.No. & Stromal changes - 35/157 & No. of cases / 157 \\
\hline 1 & Hyalinization & $25(15.92 \%)$ \\
\hline 2 & PASH like Stroma & $3(1.91 \%)$ \\
\hline 3 & Elastosis & $5(3.18 \%)$ \\
\hline 4 & Stromal Edema & $1(0.63 \%)$ \\
\hline 5 & Fat Necrosis & $3(1.91 \%)$ \\
\hline \multicolumn{2}{|c|}{ Other Changes - 45/157 } \\
\hline 1 & $\begin{array}{l}\text { Lymphatic and vascular } \\
\text { invasion }\end{array}$ & $12(7.64 \%)$ \\
\hline 2 & Periductal Inflammation & $20(12.73 \%)$ \\
\hline 3 & Giant Cell Reaction & $7(4.45 \%)$ \\
\hline 4 & Calcification & $9(5.73 \%)$ \\
\hline 5 & Medial Calcification & $8(5.09 \%)$ \\
\hline 6 & Granulation tissue & $2(1.27 \%)$ \\
\hline 7 & Thrombosed vessels & $1(0.63 \%)$ \\
\hline
\end{tabular}


Fig. 1: Diagram of gross specimen showing sections from surrounding tissue in; A) Wide local excision; B) Mastectomy Specimen; C) Cut section of mastectomy specimen showing a well circumscribed tumour mass (arrow) and surrounding whitish breast tissue
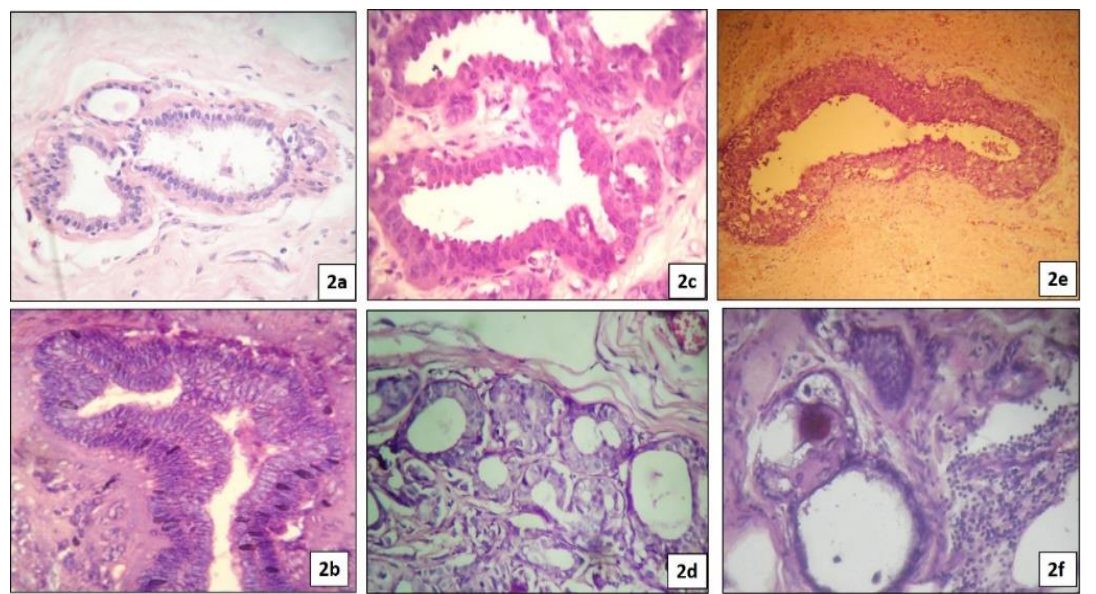

Fig. 2: Morphological Spectrum of columnar cell lesions; 2A) Columnar cell change; 2B) Columnar cell hyperplasia; 2C) Columnar cell change with atypia; 2D) Columnar cell hyperplasia with atypia; 2E) Flat epithelial atypia; 2F) Intraluminal microcalcification 

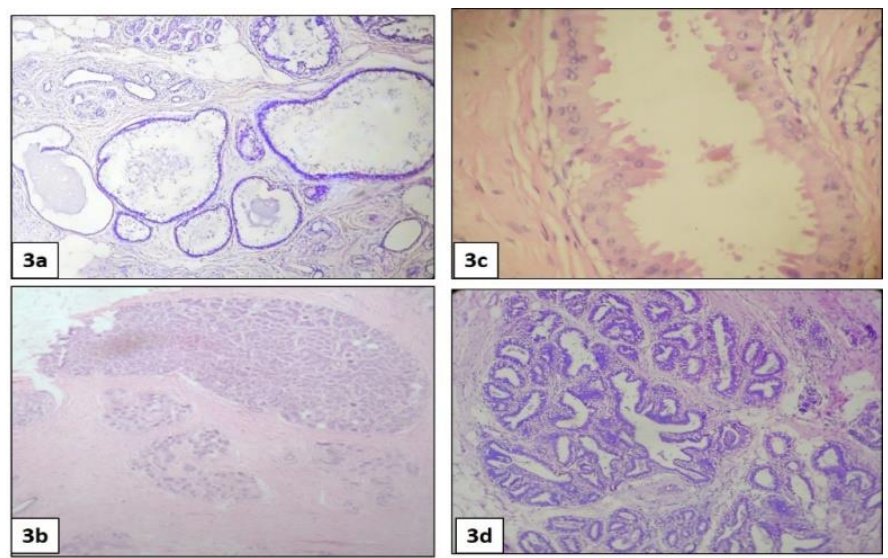

Fig. 3: Epithelial changes in surrounding breast tissue; 3A) Cystic change; 3B) Hyperplastic lobule; 3C) Apocrine cystic change; 3D) Blunt duct adenosis

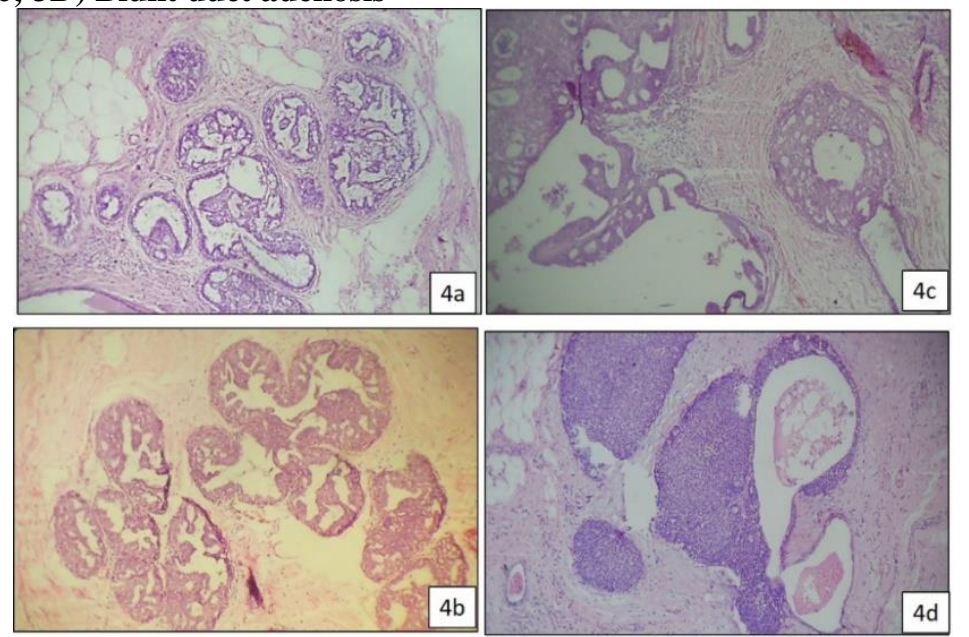

Fig. 4: Spectrum of epithelial hyperplasia in surrounding breast tissue; 4A) Usual ductal hyperplasia; 4B) Atypical ductal hyperplasia; 4C) Ductal carcinoma in situ (DCIS) micropapillary and cribriform type; 4D) Solid DCIS

\section{Conclusion}

Molecular studies have proved the association between FEA and invasive breast cancer. Early diagnosis of breast cancer depends on the detection of precursor lesions like ADH, DCIS and FEA (DIN Ia). A diagnosis of FEA is primarily a cytological one, requiring medium-power to high power microscopic evaluation to recognize the presence of low-grade cytological atypia. Architectural features do not play a significant role in the diagnosis of FEA as they do in ADH and other types of DCIS; hence can be missed easily on low power. Diagnostic accuracy can be achieved by getting better acquainted morphologically with these lesions by studying them frequently in breast tissue surrounding the lesions in surgical pathology specimens, since this is an easily available source for such lesions. These tissue sections which otherwise are a part of wastage material can thus form a good source of study material for spectrum of breast lesions. Other morphological changes in the stroma can also be studied in such sections.

\section{Conflict of Interest: None}

\section{References}

1. Lakhani SR, Ellis IO, Schnitt SJ, Tan PH., van de Vijver MJ, WHO classification of tumors of the breast. IARC Lyon 4th edition, 2012.

2. Straux P. Detection of breast cancer. Cancer 1990;66:1336-40.

3. Moinfar F. Flat ductal Intraepithelial Neoplasia of the breast: a review of diagnostic criteria, differential diagnoses, Molecular-Genetic Findings, and Clinical Relevance- it is time to appreciate the Azzopardi Concept. Arch Pathol Lab Med 2009;133(6):879-92.

4. Allen DC, Cameron RL, Histopathology Specimens m Clinical, Pathological and laboratory aspects, $1^{\text {nd }}$ edition 2004, springer.

5. Turashvili G, Hayes M, Gilks B, Watson P, Aparicio S. Are columnar cell lesions the earliest histologically detectable non-obligate precursor of breast cancer. Virchows Arch 2008;452:589-98.

6. Bloodgood J. Senile Parenchymatous hypertrophy of female breast. Its relation to cyst formation and carcinoma. Surg Gynecol Obstet. 1906;3:72130. Problems in Breast Pathology. Philadelphia: WB Saunders; 1979.5 
7. Rosen PP. Rosen's Breast Pathology. Brogi. $2^{\text {nd }}$ edition. Philadelphia, PA, Lippincott Williams and Wilkins. Philadelphia; 2001.

8. Schnitt SJ, The diagnosis and management of preinvasive breast disease: Flat epithelial atypia classification, pathologic features and clinical significance. Breast Cancer Res 2003;5(5):263-68.

9. Pinder SE, Reis-Filho J S. Non-operative breast pathology: Columnar cell lesions. J Clin Pathol 2007;60:1307-12.

10. Schnitt SJ, Vincent-Salomen A. Columnar Cell Lesions of the breast. Adv Anat Pathol. 2003;10(3):113-24.

11. Walker RA, Hanby A, Pinder SE, Thomas J, Ellis IO. Current issues in diagnostic breast pathology. J Clin Pathol 2012; 65:771-85.

12. Allred DC, Mohsin SK, Fuqua SA. Histological and biological evolution of human premalignant breast disease. Endocr Relat Cancer 2001;8(1):47-61.

13. Azzopardi JG. Problems in Breast Pathology. London, England; WB Saunders Co Ltd; 1979:193-203

14. Tan PH, HO B C-S, Selvarajan, Yap WM, Hanby A. Pathological diagnosis of columnar cell lesions of the breast. Are there issues of reproducibility?. Clin Pathol 2005;58:705-9.

15. Tavassoli FA, Hoefler H, Rosai J. Intraductal proliferative lesions. In: Tavassoli FA, Devilee P, eds, Pathology and genetics of tumors of the breast and Female genital organs. Lyon, France: IARC Press; 2003, World Health Organization Classification of Tumors

16. Pathmanathan N, Salibury EL, Provan PJ, Bilous MA, Byth K, Milliken JS et al. A class discovery and class prediction approach to histopathological classification of mammographic screen detected columnar cell lesions of the breast. Pathol 2010;42(1):28-36

17. Lerwill F. Flat epithelial Atypia of the breast. Arch Pathol Lab Med 2008;132:615-21.

18. Zagouri F, Sergentanis TN, Zografos GC. Precursors and preinvasive lesions of the breast: the role of molecular prognostic markers in the diagnostic and therapeutic dilemma. World J Surg Oncol 2007;5:57.

19. Pinder SE, Ellis IO. The diagnosis and management of preinvasive breast disease: ductal carcinoma in situ and atypical ductal hyperplasia (ADH)-current definitions and classification. Breast Cancer Res 2003;5:254-57.

20. Costarelli L, Campagna D, Mauri M, Fortunato L. Intraductal proliferative lesions of the breast- terminology and biology matter: premalignant Lesions or preinvasive Cancer?. Int J Surg Oncol. 2012;2012:501904.

21. Bombonati A, Sgroi. The molecular Pathology of Breast Cancer Progression. J Pathol 2011;223:307-17

22. Jacobs TW, Pliss N, Kouria G. Carcinomas in situ of the breast with indeterminable features: role of E-cadherin staining in categorization. Am J Surg Pathol 2001;25:229-36.

How to cite the article: Bhat D, Malani P. A, Kumbhalkar D.T. Learning from the wastage: histomorphology of columnar cell lesions and other changes in surgical pathology breast specimens. Arch Cytol Histopathol Res 2018;3(4):173-180. 